\title{
Bulk production of graphene by innovative milling techniques
}

\author{
P.S. Mukherjee* and K. Jayasankar \\ Advanced Materials Technology Department \\ CSIR-Institute of Minerals \& Materials Technology \\ Bhubaneswar - 751013, India. \\ *Email: psmukherjee52@gmail.com
}

The unique properties of graphene-based derivatives have attracted numerous research fields in recent years. The reliable and mass production of high-quality graphene is still a challenging task. This work represents the bulk production of high quality grapheme by shear force dominated dual drive ball milling of graphite with oxalic acid. The thickness of the graphene sheets was controlled by the optimized critical speed (40\%) and milling hours $(20 \mathrm{~h})$. Low diameter stainless steel balls were utilized to increase the impact and shear energy between the balls and powder, and to have a low dimensional interaction with the graphite layers. Dual-drive ball milling (Figure 1) can produce $50 \mathrm{~g}$ of graphene powder (in a single jar) and $200 \mathrm{~g}$ of graphene powder in a unique milling cycle (mechanism: Figure 2). The main advantages of dual-drive ball milling compared to other graphene preparation techniques are the simple process, cost effectiveness, and large-scale production. The introduction of metal impurities on the surface of the graphene sheets from the balls, the energy gradient between the balls and powder are the major issue in this technique. Various aspects of this process and a detailed characterization of the grapheme including probable mechanism will be discussed.

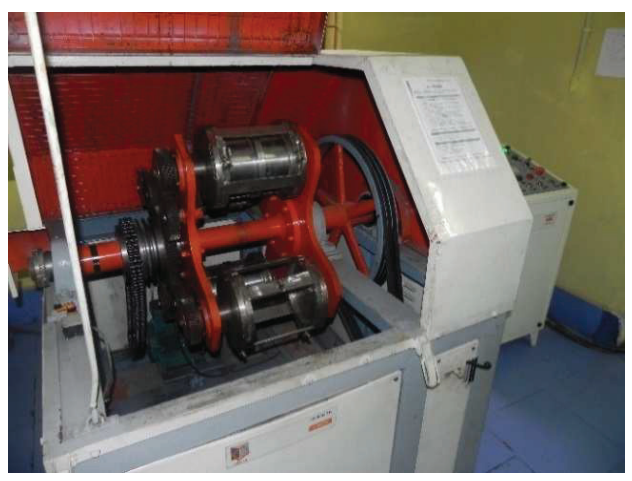

Figure 1: Dual-drive ball milling

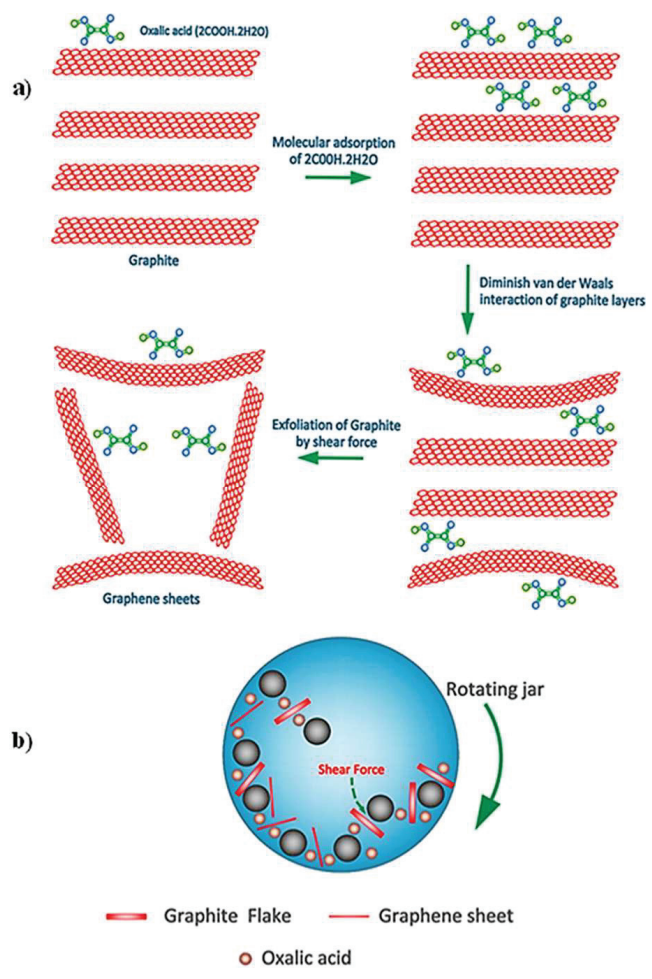

Figure 2: (a) Mechanism of graphite exfoliation through shear-force ball milling and (b) schematic representation of the ball milling of graphite with oxalic acid [1]

\section{Reference}

1. G. Rajendra Kumar et al., RSC Adv., 2016, 6, 20067-20073 